\title{
La práctica de las habilidades lingüísticas del idioma inglés en relación con el rendimiento de la prueba diagnóstica TOEFL
}

\author{
The practice of the linguistic abilities in English in relation \\ to the performance in the TOEFL diagnostic test
}

Mauricio Leonel Alvarado' Universidad Tecnológica de El Salvador mauricio.alvarado@utec.edu.sv

Recibido: 20/04/18 - Aceptado: 7/05/18

URI: http://hdl.handle.net/11298/450

DOI: http://dx.doi.org/10.5377/entorno.v0i65.6047

\section{Resumen}

Este artículo es el resultado de una investigación realizada con el objetivo de identificar las prácticas de los estudiantes inscritos en la asignatura Estrategias de Evaluación de la carrera de Idioma Inglés, de la Universidad Tecnológica de El Salvador, y también identificar el puntaje obtenido en una prueba diagnóstica de la Prueba de Inglés como Lengua Extranjera (TOEFL, siglas en inglés) test, y de esta manera describir la práctica diaria de las habilidades en el idioma inglés, como la lectura comprensiva, escritura y comprensión auditiva en diferentes actividades y lugares; y su vinculación con el desempeño en la prueba.

\section{Palabras clave}

Mediciones y pruebas educativas - Estadísticas. Lenguaje y lenguas - Pruebas de aptitud. Competencia en educación. Inglés - Enseñanza Superior.

\section{Abstract}

This article is the result of a research conducted with the objective of identifying the practices of the students enrolled in the subject Evaluation Strategies, a required course for the English Major at Universidad Tecnológica de El Salvador. It also aimed at identifying the scores obtained in the Test of English as a Foreign Languages (TOEFL) diagnostic text. In doing so, this would facilitate the description of the daily practices of the abilities in the English language such as reading comprehension, writing and listening comprehension when performing different activities, at different places, and their relation to the students ${ }^{\prime}$ performance in the test.

\section{Keywords}

Measurements and educational tests-Statistics. Language and languages-Aptitude Tests. Competence in education. English. Higher Education. 


\section{Introducción}

Comúnmente y sin hacer ningún tipo de estudio, se presume que un estudiante inscrito en un programa determinado de estudio de un idioma deberá alcanzar ciertas competencias que le permitan desempeñarse según lo previsto en los objetivos del programa. De esta manera, si se realizara una prueba estandarizada al concluir los diferentes niveles, los resultados deberían ser más o menos homogéneos; sin embargo, la realidad no siempre concuerda con este planteamiento y presunción de cómo se desenvuelven estudiantes que hayan cursado un programa de inglés.

Existen diversas prácticas que están sugeridas para lograr un novel dominio de una lengua extranjera que permita definir a un usuario de esa lengua como exitoso. También es importante resaltar que no todos los estudiantes asumen la responsabilidad de aprender con el mismo interés y dedicación. De ahí que surja la necesidad de determinar cuáles son las prácticas de las habilidades lingüísticas del idioma inglés, adquiridas por los estudiantes del quinto año de la carrera Licenciatura en Idioma Inglés, cursando la materia de Estrategias de Evaluación durante el primer ciclo del año 2017, para intentar vincularlas con en el resultado de la Prueba de Inglés como Lengua Extranjera (TOEFL, siglas del inglés), que diagnostica sus competencias en dicho idioma, realizada al inicio del ciclo.

\section{Habilidades de un segundo idioma}

Las habilidades para dominar un idioma son las que permiten comunicarse apropiadamente con otros hablantes (Harper, 2017).

Cuando se aprende un idioma, se deben desarrollar homogéneamente cuatro macrohabilidades para establecer una comunicación efectiva y para lograr un desarrollo de competencias lingüísticas de manera integral: comprensión auditiva, expresión oral, lectura y escritura.

\section{Comprensión auditiva}

Para llegar a ser un hablante del idioma inglés, es necesario desarrollar una sólida comprensión auditiva, que permita no solo comprender lo que otros digan, sino también ha- blar de manera clara,. El desarrollo de esta destreza permite aprender a pronunciar palabras adecuadamente, a utilizar una correcta entonación y a acentuar los vocablos de manera acertada, lo que hace que otros comprendan de manera precisa lo que se dice (Buswell, 1997).

\section{Expresión oral}

Se define como el proceso empleado para expresar ideas, ya sea de manera personal o por otros medios que permitan la comunicación de forma no presencial.

\section{Lectura}

La lectura es un proceso de decodificación de textos escritos que requiere la utilización de conocimiento de un idioma. Este proceso incluye conocer vocabulario, organización, estilo e intención del texto. En el caso del inglés, es necesario conocer los patrones de escritura más comunes.

\section{Escritura}

Para escribir en un segundo idioma, es esencial entender el sistema básico de ese idioma, y a partir de ese punto, elaborar y recombinar estructuras que permitan transmitir un mensaje de manera efectiva. En inglés, esto incluye el conocimiento de ortografía y gramática.

TOEFL test

Desde finales de la década de los 60, la Prueba de Inglés como Lengua Extranjera (TOEFL, siglas en inglés) ha sido administrada por Educational Testing Service $(E T S \circledR)$, una organización responsable de administrar test estandarizados. Entre 1964 y 2008, de acuerdo con esta institución, 24 millones de personas, principalmente estudiantes internacionales, han tomado el test.

\section{Estructura del TOEFL}

Desde 1988, el test escrito ha estado constituido de 50 preguntas basadas en comprensión auditiva, 40 de estructura y expresión escrita y 50 de lectura comprensiva.

La siguiente tabla ilustra la distribución de las secciones del test. 
Tabla 1.

\begin{tabular}{llrr}
\hline \multicolumn{1}{c}{ SECCIÓN } & \multicolumn{1}{c}{ DESCRIPCIÓN } & No. DE PREGUNTAS & TIEMPO \\
\hline Comprensión auditiva & Mide la habilidad para entender inglés hablado & 50 & $30-40$ min \\
Estructura y expresión escrita & Mide la habilidad para el inglés escrito estandarizado & 40 & 25 min \\
Comprensión lectora & Mide la habilidad para leer material no técnico & 50 & 55 min \\
\hline
\end{tabular}

\section{Metodología}

Se empleó el método cuantitativo para la obtención de los datos y se realizó un estudio no experimental.

La población de este estudio estuvo constituida por 82 estudiantes de quinto año de la Licenciatura en Idioma Inglés inscritos en la asignatura Estrategias de Evaluación, de la Universidad Tecnológica de El Salvador.
Los instrumentos utilizados fueron el cuestionario y una prueba diagnóstica del examen TOEFL con todos los componentes de una prueba estandarizada TOEFL realizada bajo condiciones que emulan la administración de la prueba oficialmente administrada por las instituciones autorizadas por ETS $®$ (Educational Testing Service), institución reguladora de la creación y administración del TOEFL test a nivel mundial.

\section{Resultados}

Figura 1. Resultados de la administración de la prueba diagnóstica

\section{Diagnostic TOEFL Test}

\section{0-300 $\square$ 301-400 $\square$ 401-499 $\square$ 500+}

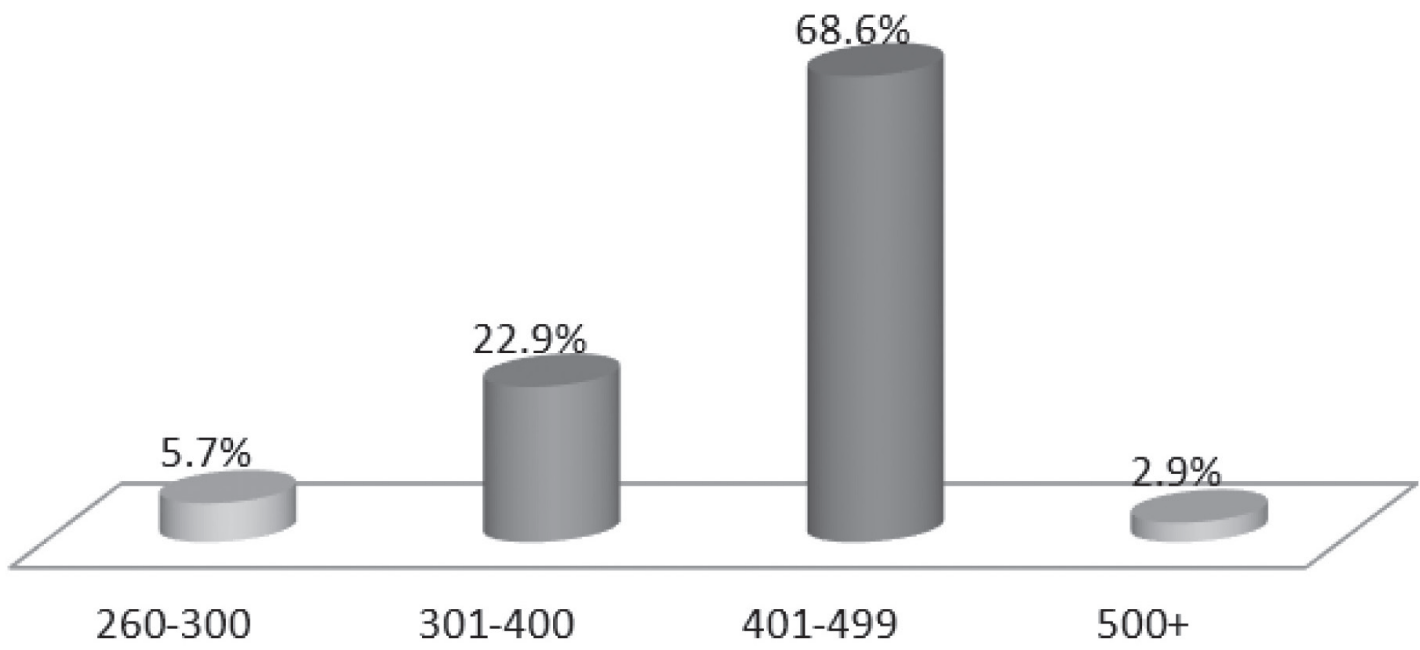

La prueba tiene un puntaje máximo de 677, de esos, menos del $3 \%$ de los estudiantes que realizaron la prueba diagnóstica del TOEFL alcanzaron el puntaje más alto (513), que se registró en la administración de esa prueba en la asignatura de Estrategias de Evaluación. El 68,6 \% de los examinados se encuentran en un promedio que comprende entre $401 \mathrm{y}$
499 puntos en el TOEFL, siendo 500 puntos el mínimo establecido por la universidad para poder graduarse. Este grupo de estudiantes está en un rango no apto para graduarse por no haber alcanzado este puntaje. Luego, en el extremo más bajo de la tabla se encuentran los que obtuvieron puntajes por debajo de lo requerido por la universidad como requisi- 
to de graduación, resultando el 22,9 \% en un rango de 301 a 400 puntos, y 5,7\%, entre 260 a 300. Es de resaltar que las últimas dos agrupaciones de puntajes representan resultados muy bajos, en cuanto al dominio del idioma inglés, debi- do a que el puntaje menor, en caso que un obtuviese todas las respuestas incorrectas, es de 217 puntos, según la tabla de calificación establecida por la entidad responsable de regular la creación y administración del TOEFL Test ETS $®$.

Figura 2. Práctica de inglés de los estudiantes

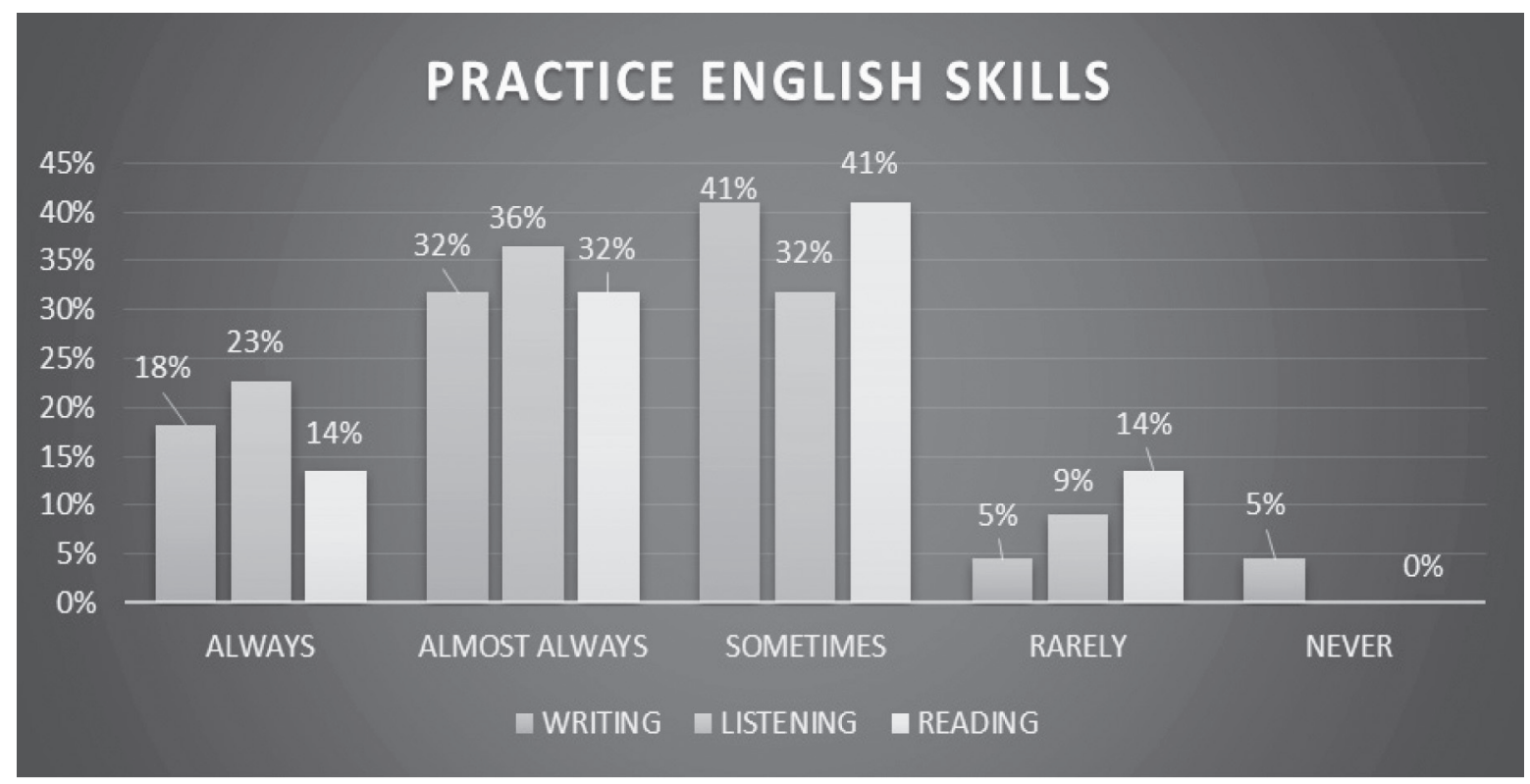

En cuanto a comprensión auditiva realizada, $23 \%$ de los encuestados manifestaron que la frecuencia con que ejercitan su destreza de comprensión auditiva es siempre, mientras que un 36 \% casi siempre practica la comprensión auditiva como parte de su estrategia de estudio o práctica del idioma inglés, para el desarrollo de competencias lingüísticas. El 32 \% de los encuestados están en una frecuencia de algunas veces en cuanto a la práctica que realizan de la comprensión auditiva para desarrollar esa área de dominio del idioma.

Por otra parte, en la práctica de la lectura, el 14 \% de los encuestados manifestaron que siempre realizan prácticas que les ayuden a desarrollar la comprensión lectora. Mientras que quienes casi siempre practican la lectura en el idioma inglés alcanzan el $32 \%$ de los encuestados.

El otro componente evaluado en el TOEFL es conocimiento de la estructura del idioma inglés, y para ello se vinculó con la escritura debido a que es la práctica de esta donde los estudiantes deben aplicar el conocimiento de la estructura y las reglas gramaticales del idioma. En ese sentido, el $18 \%$ de los encuestados incluyeron la escritura dentro de su práctica para desarrollar competencias en el idioma inglés. El 32 \% casi siempre escribe, como práctica del idioma inglés, en su búsqueda por mejorar su dominio. El $41 \%$ de los encuestados realiza una práctica con una frecuencia de algunas veces para lograr un dominio del idioma inglés.

\section{Consideraciones}

- $50 \%$ de los estudiantes manifestaron que una de las formas más frecuentes que emplean para practicar la habilidad de comprensión auditiva es escuchando música.

- Se incluyó una pregunta para determinar si los estudiantes trabajaban o no, y $27,2 \%$ de los estudiantes que trabajan han tomado cursos de inglés adicionales.

- Los estudiantes que trabajan tienen más conocimiento acerca de la estructura del examen con respecto a quienes no trabajan. 
- Las mujeres manifestaron tener mayores dificultades con el vocabulario en inglés.

- $50 \%$ de los estudiantes encuestados manifestaron tener dificultad en el área de las estructuras gramaticales del examen.

\section{Referencias}

Barake, F.H. (2012). "Reglamento especial para el funcionamiento de carreras y cursos que habilitan para el ejercicio de la docencia en El Salvador". San Salvador: Dirección Nacional de Educación Superior.

Barry, L. (2009). Teaching English. Recuperado el de marzo de 2017, de https://www.teachingenglish.org.uk/article/ proficiency-test

Buswell, N. (1997). English Club. Recuperado el 15 de marzo de 2017, de https://www.englishclub.com/learnenglish/language-skills.htm

Calderón, M. (2013). Reading Comprehension Skills for English Language Learners. Colorin Colorado.
Cambridge. (2017). Recuperado el 17 de marzo de 2017, de http://www.examenglish.com/CEFR/cefr.php

Cambridge. (2017). Cambridge English. Recuperado el 17th de March de 2017, de http://www.cambridgeenglish. org/exams/cefr/

ETS (2017). ETS. Recuperado el 17 de marzo de 2017, de https://www.ets.org/toefl/ibt/about/content/

Harper Collins. (17 de marzo de 2017). CollinsDictionary. com. Obtenido de Free Online Dictionary: https:// www.collinsdictionary.com/dictionary/english/skill

Rogers, B. (2011). The Complete Guide to the TOEFL Test: PBT Edition. Boston, Massachussetts, USA: Heinle Cengage Learning.

Swarthout, D. (2003). Study.com. Recuperado el 17 de marzo de 2017, de http://study.com/academy/lesson/oralcommunication-definition-types-advantages.html

Woodley (2017). Reference. Recuperado el 17 de marzo de 2017, de https://www.reference.com/education/ diagnostic-test-education-53d1574cddd87aca\# 